ESAIM: PROCEEDINGS, July 2007, Vol.18, 87-98

Jean-Frédéric Gerbeau \& Stéphane Labbé, Editors

\title{
DISCUSSION OF A SIMPLE MODEL OF SPREADING DEPRESSIONS*
}

\author{
Guillemette Chapuisat ${ }^{1}$
}

\begin{abstract}
This paper is devoted to the study of a model of spreading depression (SD) which is a profound and transient depolarization of neurons. SD have a great importance in stroke or migraine with aura for example. Contrary to the rodent brain, SD have never been observed in the human brain. An explanation of this difference is proposed here by studying the influence of the geometry and composition of the brain.

The model is derived from experimental observations and leads to a reaction-diffusion system that governs the ionic exchanges in calcium and potassium between the various cells of the brain and the extracellular space. Parameters of the model are fixed using a random algorithm that is explained here. Then the influence of geometry and composition of the brain is numerically studied.
\end{abstract}

Résumé. Les dépressions corticales envahissantes (DCE) sont des ondes de dépolarisation temporaire des neurones qui se propagent dans le cerveau à la suite d'une migraine avec aura, d'une crise d'épilepsie ou d'un accident vasculaire cérébral. Ces DCE sont connues depuis longtemps dans le cerveau du rongeur, mais n'ont pour l'instant jamais été observées chez l'homme. C'est pour essayer de comprendre la non-observation de ces ondes chez l'homme que nous avons développé un modèle mathématique de DCE.

Les DCE sont liées à des concentrations ioniques anormales dans les diverses cellules du cerveau et dans l'espace extracellulaire. En suivant les observations de Nedergaard [3], nous avons construit un modèle simple de DCE basé sur les échanges ioniques en calcium et en potassium entre les différents compartiments du cerveau. On obtient un système de réaction-diffusion.

Un problème est alors de déterminer les valeurs des paramètres du système. Les expériences étant souvent impossibles ou soumises à de fortes marges d'erreur, nous avons décidé d'utiliser un algorithme qui tire au hasard les paramètres du système, puis qui conserve les paramètres en mémoire si certaines propriétés qualitatives de la solution du modèle sont vérifiées.

Une fois le modèle établi et validé, nous avons pu tester l'effet des différences de géométrie et de composition des cerveaux sur les DCE. En effet, le cerveau du rongeur est lisse et intégralement composé de matière grise (zone où se trouvent les corps des neurones), alors que le cerveau de l'homme a de nombreuses circonvolutions et est principalement composé de matière blanche avec une fine couche de matière grise à la périphérie. Comme la matière blanche ne contient pas de neurone, le modèle affirme que l'étape de réaction n'a pas lieu dans cette matière, les DCE y diffusent simplement et sont progressivement absorbées. On constate alors que si la couche de matière grise est fine, l'effet absorbant de la matière blanche empêche la création de DCE. D'autre part, une DCE stoppe sa progression à la première forte et brusque augmentation de l'épaisseur de la matière grise. Ainsi les différences de structure entre les cerveaux pourraient à elles-seules expliquer la non-observation des DCE.

\footnotetext{
* This work was supported by the French Ministry of Research (ACI "AVC-iSi", project No 02TS041) and developped with the Institut de Médecine Théorique chaired by J.P. Boissel.

${ }^{1}$ Laboratoire de Mathématiques, Université Paris-Sud XI, Bât. 425, 91405 Orsay Cédex. e-mail: guillemette.chapuisat@math.u-psud.fr
}

(C) EDP Sciences, SMAI 2007 


\section{INTRODUCTION}

Spreading depressions (SD) consist of a transient suppression of all neuronal activities [12]. This suppression spreads slowly across a large region of the brain. They were first observed and described in 1944 by Leão in [9]. SD play most likely an important role in many diseases for example in migraine with aura, epilepsy and cerebral stroke. They have been observed in many species, but their existence is still discussed in the human cortex. For the moment, they have never been experimentally observed in human brain, perhaps because of the difficulties of in vivo measurements.

It is very important to understand the phenomena that are responsible for the creation or extinction of SD [8]. Indeed in cerebral strokes in rats, the number of SD has a clear influence on the size of the final infarcted area (i.e. area of the brain where all the cells are dead). Some chemicals that block the appearance of SD have already been studied and reduce importantly after-effects of strokes in the rat brain. Unfortunately, they seem to be ineffective on humans. Mathematical models of SD may help to explain this difference [5,7,12-14].

The aim of this paper is to present a model of SD based on the experimental observations of Nedergaard in [3]. SD are linked to ionic exchanges between the various cells that compose the brain and the extracellular space.

Indeed brain is composed of several types of cells. Neurons convey the electrical informations. They form a network which is surrounded by all the other cells called glial cells. Astrocytes are part of these glial cells. They also form a network. The role of astrocytes is mainly to maintain a favourable environment for neurons.

To convey electrical informations, the neurons need to be polarized. This polarization is due to a differential in ionic concentrations between intra- and extra-cellular spaces. For example, the potassium concentration is markedly higher in the neurons than in the extracellular space. It is the opposite for the calcium concentration. A cell has various possibilities to maintain this differential between intra- and extra-cellular concentration. A first possibility is to use ionic pumps. These pumps make a ionic species go through the cell's membrane in a specific sense. Their functioning depends on the other ionic concentrations. Moreover these pumps need energy to work. The second possibility for ions to go through the cell's membrane is gap junctions. A gap junction can be considered as a hole between two cells that lets only a specific ionic species go through it.

It has been shown that SD are triggered by a large increase of the extracellular potassium concentration. Besides SD are characterized by a potassium wave that propagates through the extracellular space. Moreover during $\mathrm{SD}$, a calcium wave through the astrocytes has been experimentally observed. Thus the model is centered around the ionic exchanges of potassium and calcium between neurons, astrocytes and extracellular space.

The detail of the model will be given in the next section. Then in section 3 , we will discuss the values of all the parameters involved in this model. Finally in section 4, we will explain the results of this model, that means the new phenomena which appear when we combine all the elementary points, but which are not programmed in the model.

\section{MAin FEATURES OF THE MODEL OF SD}

In this model, we will consider three different spaces in the brain: the extracellular space, the neural network and the astrocytic network. Each space will be supposed continuous. We will take into account two cations: $\mathrm{Ca}^{2+}$ and $\mathrm{K}^{+}$.

SD look like propagative waves and as in many biological situations [10], Nedergaard's observations lead indeed to a reaction-diffusion system.

The basic phenomena that create spreading depressions are as follow:

- The potassium ions can diffuse in the extracellular space.

- An excess of potassium in the extracellular space activates ionic pumps on the astrocytes' membrane that make calcium enter the cell.

- The calcium diffuses through gap junctions in the astrocytic network.

- The calcium ions enter the neurons through gap junctions between neurons and astrocytes. 
- The excess of calcium in the neuronal space activates ionic pumps on the neurons' membrane that make potassium go out of the cell.

All the preceding basic phenomena make ionic concentrations increase. We now have to add some recovery mechanisms in order to make the system go back to his rest state. First small variations in the ionic concentrations are quickly erased by various channels (ionic pumps, ...). If the variations are too large, then another mechanism takes place and tries to limit these variations. Few biological informations are available on this mechanism. At last there is a special phenomenon to recover from spreading depression which is expressed by a very important increase of calcium and potassium concentrations. This recovery from SD is either not perfectly understood, but at least we know that it needs several steps (in particular, a blood flow increase). This implies a delay before the cell is ready to recover.

Nedergaard's observations will permit us to construct a mathematical model of SD that can be quickly numerically solved. This point is important since we want then to integer this model in a global model of stroke. For example, Shapiro's model in [11] is very complete, but really too heavy in order to be integrated. Another interest of this the model is that it is really based on some biophysical membrane mechanisms which was not the case in [13]. Finally, this model takes into account the propagation of SD unlike Somjen's model in [12] which is only valid for a single neuron. This model will be phenomenological which means that the links between the various variables are based on biophysical observations but qualitatively established.

Let us call $C a_{g}$ the variation of calcium concentration in the astrocytes ( $g$ stands for glia) in relation to the rest state. So $C a_{g}$ equals 0 when the calcium concentration in the astrocytes is normal. In the same way, we call $C a_{n}$ the variation of calcium concentration in the neurons and $K_{e}$ the variation of potassium concentration in the extracellular space.

$R$ represents the recovery mechanism from spreading depressions and Cap the capacity of the cell to recover from SD. When Cap reaches a fixed threshold, the phenomena that govern the recovery from SD can take place. We obtain the following partial differential equations.

$$
\begin{gathered}
\partial_{t} C a_{g}-\nu_{C a_{g}} \Delta C a_{g}=-k_{C a_{g}} \tanh \frac{C a_{g}}{C a_{g}^{0}}+h_{K, C a} K_{e}-k_{g a p}\left(C a_{g}-C a_{n}\right)-\alpha_{C a_{g}} C a_{g}^{2}-\alpha_{r e c, g} R C a_{g} \\
\partial_{t} C a_{n}=-k_{C a_{n}} \tanh \frac{C a_{n}}{C a_{n}^{0}}+k_{g a p}\left(C a_{g}-C a_{n}\right)-\alpha_{C a_{n}} C a_{n}^{2}-\alpha_{r e c, n} R C a_{n} \\
\partial_{t} K_{e}-\nu_{K_{e}} \Delta K_{e}=-k_{K_{e}} \tanh \frac{K_{e}}{K_{e}^{0}}+h_{C a, K} C a_{n}-\alpha_{K_{e}} K_{e}^{2}-\alpha_{r e c, e} R K_{e} \\
\partial_{t} C a p=1_{\left\{C a_{g}>c_{g}\right\} \cup\left\{C a_{n}>c_{n}\right\} \cup\left\{K_{e}>c_{k}\right\}} \frac{1-C a p}{T_{o}}-1_{\left\{C a_{g}<c_{g}\right\} \cap\left\{C a_{n}<c_{n}\right\} \cap\left\{K_{e}<c_{k}\right\}} \frac{C a p}{T_{f}} \\
\partial_{t} R=1_{\left\{C a p>c_{c a p}\right\}} \frac{1-R}{\tau_{o}}-1_{\left\{C a p<c_{c a p}\right\}} \frac{R}{\tau_{f}}
\end{gathered}
$$

The first three equations govern the ionic movements. The last two equations govern the special mechanism to recover from SD. The biological meanings of the various parameters (and thus of the different terms of the equations) are gathered in table 1.

This equations are valid in the gray matter of the brain (where there are neurons). The natural boundary condition is of homogeneous Neumann type.

\section{Discussion OF PARAMETERS}

\subsection{A problem}

In this model of SD, many parameters appear (see table 1). They have to be fixed before we can solve the system of equations. Since each part of the model represents a biological phenomenon, the first natural idea is 


\begin{tabular}{|l|l|}
\hline$\nu_{i}$ & diffusion coefficient of ion $i$ \\
\hline$k_{i}$ & strength of the recovery mechanism for small concentration variations of ion $i$ \\
\hline$C a_{n}^{0}, C a_{n}^{0}, K_{e}^{0}$ & range of the variations that are concerned with the recovery for small variations \\
\hline$\alpha_{i}$ & strength of the recovery mechanism for large variations of concentration of $i$ \\
\hline$\alpha_{r e c, i}$ & strength of the recovery from SD for ion $i$ \\
\hline$h_{K, C a}$ & $\begin{array}{l}\text { activity of the ionic pumps on astrocyte's membrane sensitive to extracellular potas- } \\
\text { sium that makes calcium enter the astrocyte. }\end{array}$ \\
\hline$k_{g a p}$ & activity of the gap junctions between astrocytes and neurons \\
\hline$h_{C a, K}$ & $\begin{array}{l}\text { activity of the ionic pumps on neuron's membrane sensitive to intracellular calcium } \\
\text { that makes potassium go out of the neuron. }\end{array}$ \\
\hline \hline$c_{i}$ & concentration thresholds above which the cell begins to prepare for the SD recovery \\
\hline$T_{o}$ & typical time for the cell to be ready to recover from SD \\
\hline$T_{f}$ & typical time for the disappearance of the preparation to recover from SD \\
\hline$c_{c a p}$ & $\begin{array}{l}\text { threshold above which the cells are able to recover from SD, connected with the } \\
\text { delay before the recovery from SD mechanism takes place }\end{array}$ \\
\hline$\tau_{o}$ & typical time for the complete opening of the recovery mechanism \\
\hline$\tau_{f}$ & typical time for the complete closure of the recovery mechanism \\
\hline
\end{tabular}

to search in the litterature for data on this phenomenon in order to obtain the different values of the parameters. Several problems will be posed.

- First, for different reasons, data are lacking (impossible experiments, no precise biological meaning ...).

- If they exist, data come from different species or different types of neurons.

- The experimental error can be very large.

So even if we have a lot of data, to fit them together requires additional work. Moreover a large system of partial differential equations with a lot of parameters can have very different behaviours and may be very sensitive to variations of one parameter. Thus we had to find another method to fix the values of the parameters.

\subsection{A basic random algorithm}

To fix the values of the parameters, the first natural idea was to use a random algorithm, i.e. choose randomly the parameters and then test whether the solution obtained with these parameters well suits the biological observations. So, we first have to decide which qualitative properties the solution of the model must have. This set of properties will be called basis of rules in the following.

Here, the basis is composed of six rules that come from experimental observations:

- 0 is a stable state.

- An injection of $\mathrm{KCl}$ with a higher concentration than $12 \mathrm{mM}$ creates one or more $\mathrm{SD}$ that are expressed by a calcium wave through the astrocytic network.

- This wave propagates with a speed about $3 \mathrm{~mm} / \mathrm{min}$.

- The increase of potassium concentration in the extracellular space is between 30 and $80 \mathrm{mM}$ and the increase of calcium concentration in the astrocytes is about $0.2 \mathrm{mM}$.

- The maximum of the potassium concentration in the extracellular space must look like a plateau.

- A continue injection of $\mathrm{KCl}$ creates successive SD.

Then the random algorithm to find the possible parameters of the model is as follows.

- Choose reasonable range for the values of all parameters.

- Choose at random the various parameters values of the set of partial differential equations.

- Test numerically whether the preceding basis of rules is satisfied. 
- If the answers of this question is positive, then keep this set of parameters in memory.

Let us remark that of course, a limit of this algorithm is that it needs a basis of rules that can be numerically tested. Moreover the method to solve the system has to be fast and efficient so that a lot of sets of parameters can be tested. Here we have decided to model the brain as a segment and to use a semi-implicite finite differences method. The problem is that the ionic concentration have rather steep variations so the time and space steps have to be small. Another important point is the choice of the reasonable range for the parameters values. The larger the space where the parameters values are chosen is, the smaller the propability of finding suitable parameters is. And since there are many parameters to find, this probability can become really tiny if the space is too large.

A $\mathrm{KCl}$ injection is simulated using the initial conditions. In a part of the brain, the initial extracellular potassium concentration is assumed higher than the rest concentration.

We have applied this procedure to the preceding model that govern ionic exchanges. It is composed of 5 equations and 27 parameters have to be fixed. At the end of a run, more than hundred thousands sets of parameters have been tested, leading to five suitable sets.

Examples of results that are kept by this algorithm are shown in figures 1 and 2. Ionic waves are created and spread through the entire brain. Every set of suitable parameters that was found with this algorithm gives similar qualitative results.

For the moment, no correlation between these suitable sets has been established, but the very small number of sets prevents us from using any statistical method.
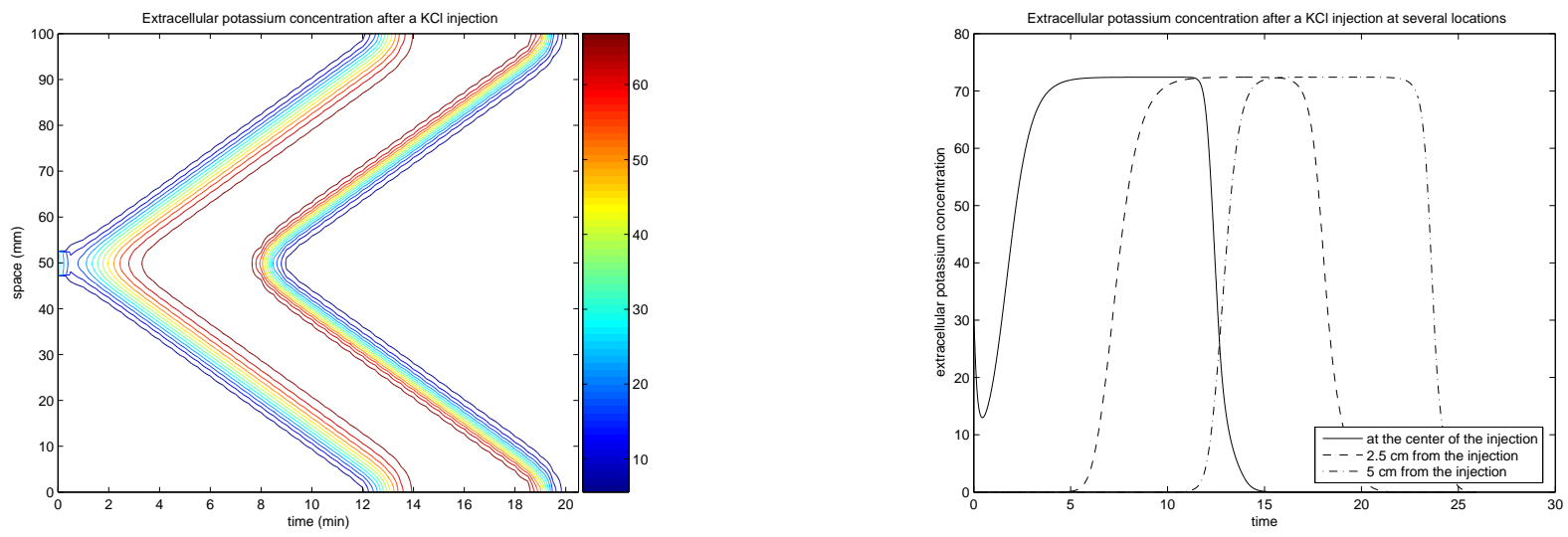

Figure 1. Potassium concentration in the extracellular space after a $\mathrm{KCl}$ injection for suitable parameters. Left, the level lines of the potassium concentration in function of the time ( $\mathrm{x}$ coordinate) and of the place in the brain (y-coordinate). Right, potassium concentration at several locations in function of time. The solid line represents the extracellular potassium concentration in the middle of the area where the injection is made. The dashed line is the potassium concentration $2.5 \mathrm{~cm}$ further and the dashed-dotted is $5 \mathrm{~cm}$ further.

In this basis of rules, an important test is the creation of successive SD for a continue injection of KCl. As a matter of fact, when a stroke occurs, successive SD are created. The reaction of the system to a continue injection for suitable parameters is shown in figure 3. In order to simulate a continue $\mathrm{KCl}$ injection, we have forced the extracellular potassium concentration to stay higher than a certain concentration $(15 \mathrm{mM})$ in part of the brain at every time step.

Another idea for fixing the values of all the parameters will be to use an algorithm where the random choice of the parameters is influenced by the preceding tested sets of parameters. In particular, we could try to use an 

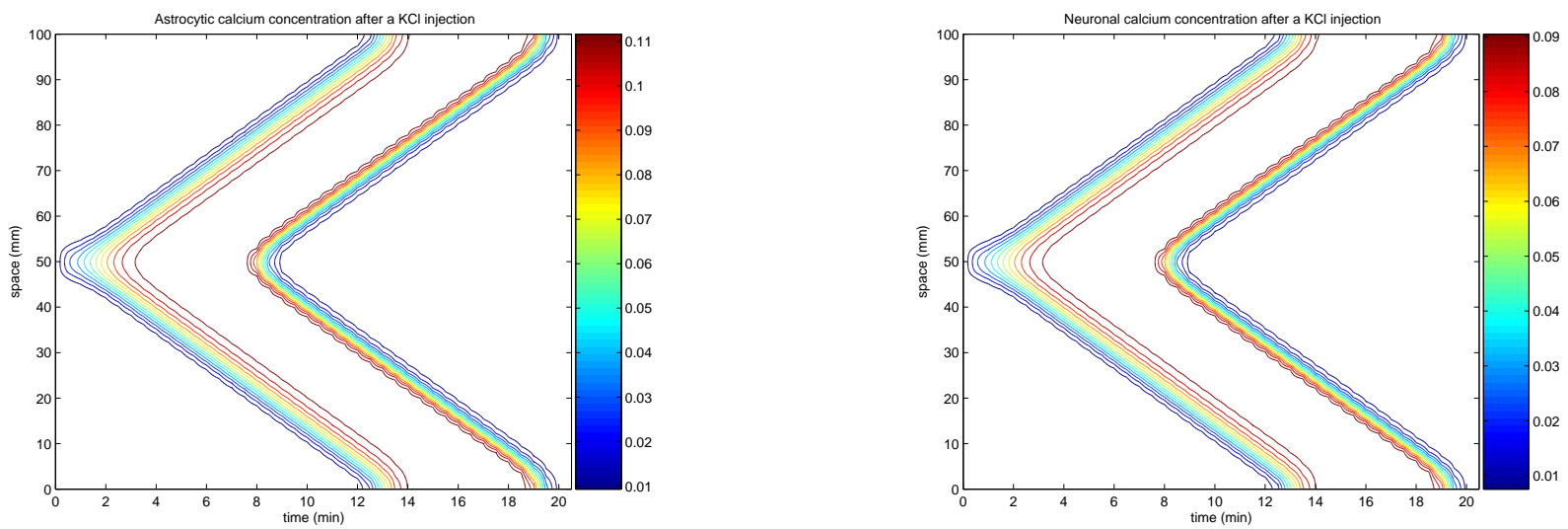

FIgURE 2. Level lines of the calcium concentration after a $\mathrm{KCl}$ injection for suitable parameters. Left, the calcium concentration in the astrocytes and right, the calcium concentration in the neurons. As it has been observed by Nedergaard, a calcium wave propagates through the network of astrocytes.

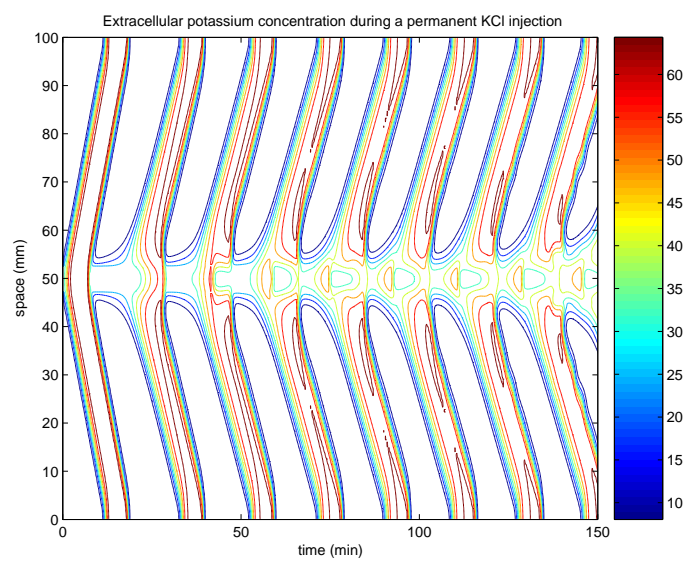

Figure 3. Potassium concentration in the extracellular space during a continue $\mathrm{KCl}$ injection for suitable parameters. Here, the brain is supposed to be a segment. Successive ionic waves are created.

evolutionary algorithm. It is a stochastic search method that tries to reproduce the natural biological evolution. In particular, it uses the principle of survival of the fittest to produce better and better approximation of a solution of a problem.

In our case, the problem was to define the "fitness" of a set of parameters. Indeed it is in function of the fitness that a set of parameters will be kept or not for the next iteration of the algorithm. Since SD can have various travelling speeds (between 2 and $6 \mathrm{~mm} / \mathrm{min}$ ) or correspond to various potassium concentration increases (between 30 and $80 \mathrm{mM}$ ), the fitness can not be defined as the distance between the solution and a fixed function. The only reasonable possibility was to define the fitness of a set of parameters as the number of tests of our basis of rules that the solution has passed. Of course you can put weights on the various tests according to their relative importance. But whatever the weights are, almost all the sets of parameters gives the same fitness and 

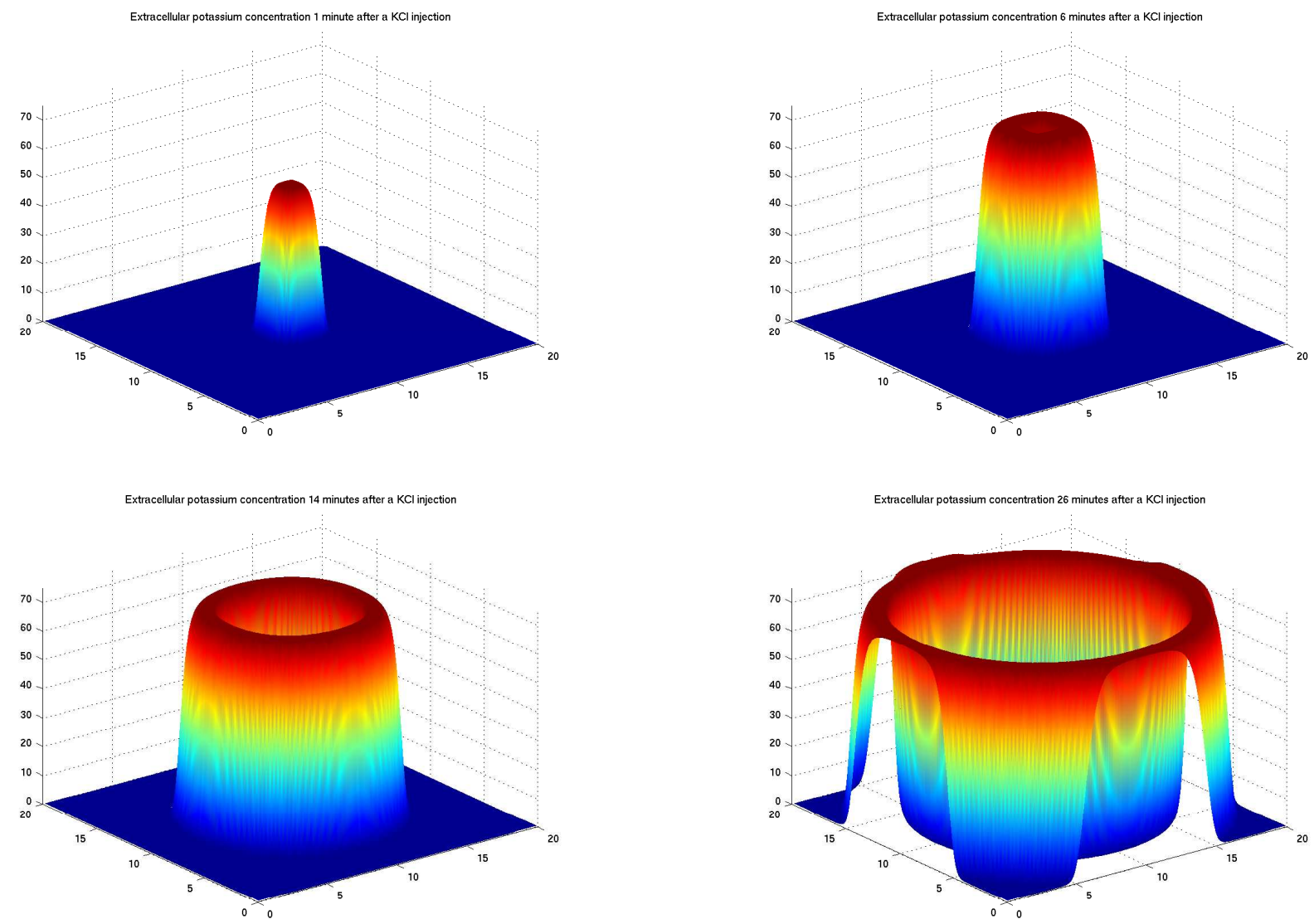

FiguRE 4. Potassium concentration in the extracellular space after a $\mathrm{KCl}$ injection for several times $(1,6,14$ and 26 min after the injection). A SD propagates through the entire brain.

so no information can be used to select the next generation of sets of parameters. Hence it appears to us quite impossible to define a fitness function that really makes sense.

We now consider that the several sets of parameters found with the random algorithm explained above suit well. Let us see what happens in other geometries.

\section{Two Dimensional RESUlts}

\subsection{Propagation of SD in the gray matter}

After having found suitable parameters for the model, we can use it to study the effect of SD in a more realistic geometry. First we decided to make the simulation in a 2 dimensions space. As a first step, the brain will be represented by a square of gray matter. Hence calculations can be done by Fast Fourier Transformation in space.

First, let us check the effect of a $\mathrm{KCl}$ injection in a normal tissue in 2 dimensions. The $\mathrm{KCl}$ injection is simulated by an initial condition with $K=20 \mathrm{mM}$ on a central square and $K=0 \mathrm{mM}$ outside. As we can see (figures 4 to 6 ), a potassium wave propagates through the extracellular space. Similarly, a calcium wave propagates through astrocytes and neurons. About 35 minutes later, the system is back to his rest state. 


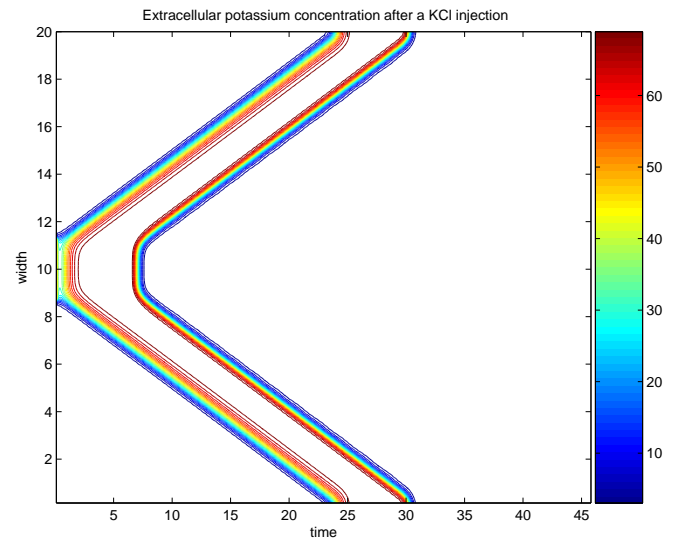

Figure 5. Potassium concentration in the extracellular space after a $\mathrm{KCl}$ injection on a diameter of the brain. Here, the brain is supposed to be a square.

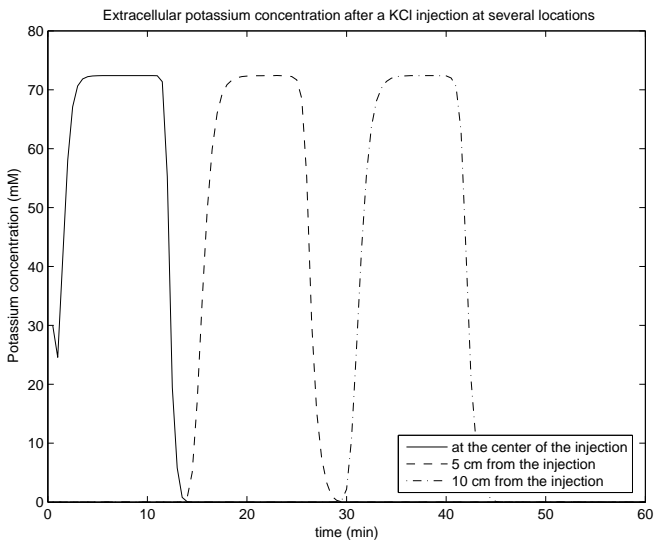

Figure 6. Extracellular potassium concentration after a $\mathrm{KCl}$ injection for several locations in the brain (center of the injection, $5 \mathrm{~cm}$ further and $10 \mathrm{~cm}$ further).

These results are consistent with the calculations made in a one dimension brain. Figure 5 shows the potassium concentration in the extracellular space for a fixed y-coordinate. We find the same profile as in section 2 .

\subsection{Influence of the white matter}

Now we will try to understand why SD have still not been observed in the human brain. Mechanisms that are responsible for the generation of SD are apparently identical in the rodent brain and in the human brain. Maybe some parameters of the model are modified in the human brain and since the system can be sensitive to some parameters, this could explain the non-observation of SD.

Another explanation may lie in the differences of geometry and composition of the rodent brain and of the human brain. Indeed a brain can eventually be composed of two different matters, the white and the gray matter. The rodent brain is smooth and entirely composed of gray matter while the human brain is mostly composed of white matter whith a thin layer of gray matter at the surface. Moreover the human brain has a very complicate geometry with a lot of circumvolutions.

In this section we will study the influence of the white matter. Indeed, SD are modeled by a reaction-diffusion system in the gray matter described above. But in the white matter, there are no neuron, so the reaction loop described by Nedergaard is interrupted. Thus the ions responsible for SD just diffuse and are progressively absorbed in the white matter. This may prevent the creation of SD. In this model, the calcium concentration in neurons $C a_{n}$ is simply set to 0 in the white matter. Moreover we assume a Neumann condition for $C a_{n}$ at the boundary of the gray matter; the other functions are assumed $C^{1}$ on the interface between the white and the gray matter.

Let us first study the effect of white matter when the layer of gray matter is thick (figure 7). SD normally spread through the entire gray matter.

Now, if the layer of gray matter is thin, we see that SD are not created despite the same initial condition as preceding (figure 8).

The fact that the creation of SD depends on the thickness of the gray matter may be an explanation for the non-observation of SD in the human brain. This numerical observation has been mathematically studied in [2]. 

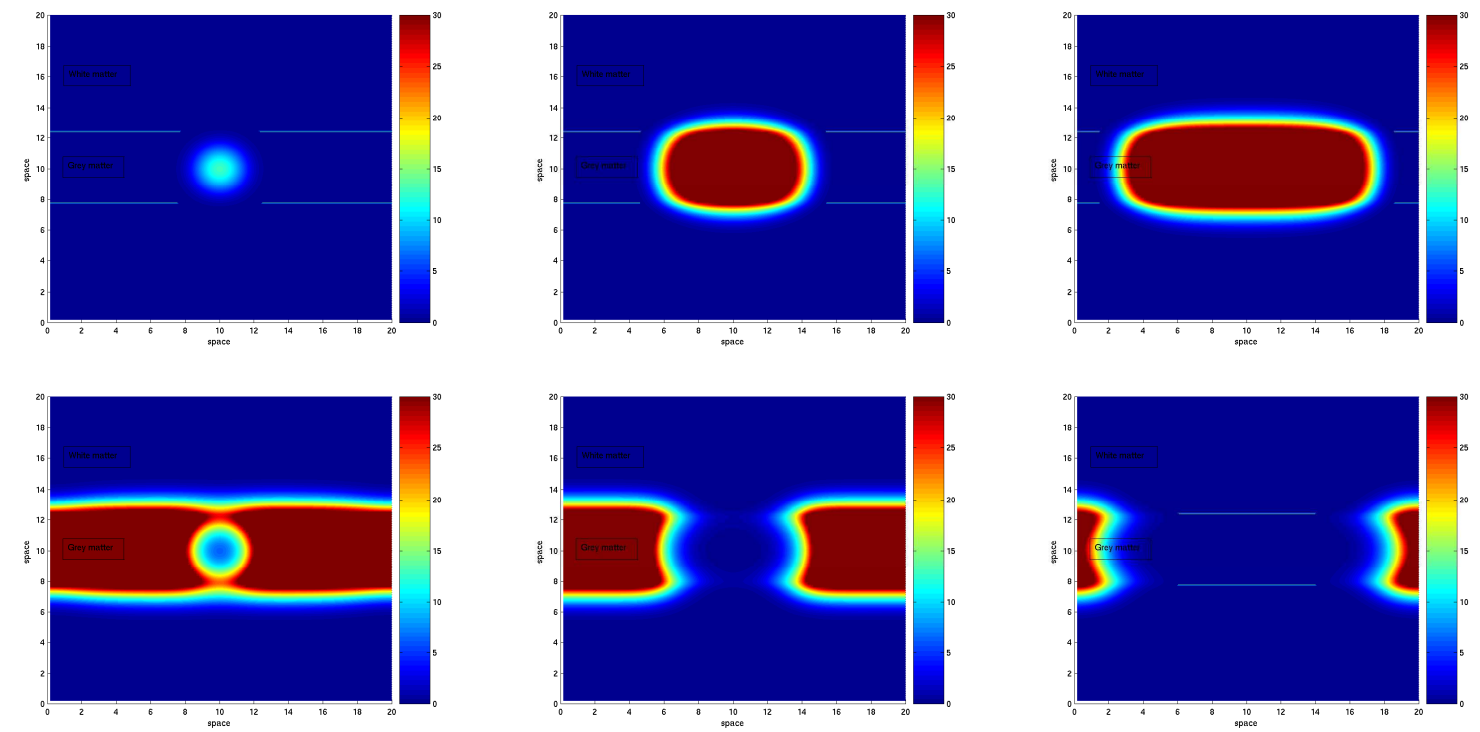

FiguRE 7. Extracellular potassium concentration after a $\mathrm{KCl}$ injection in the gray matter. Here the layer of gray matter is thick and the SD spreads through this layer.
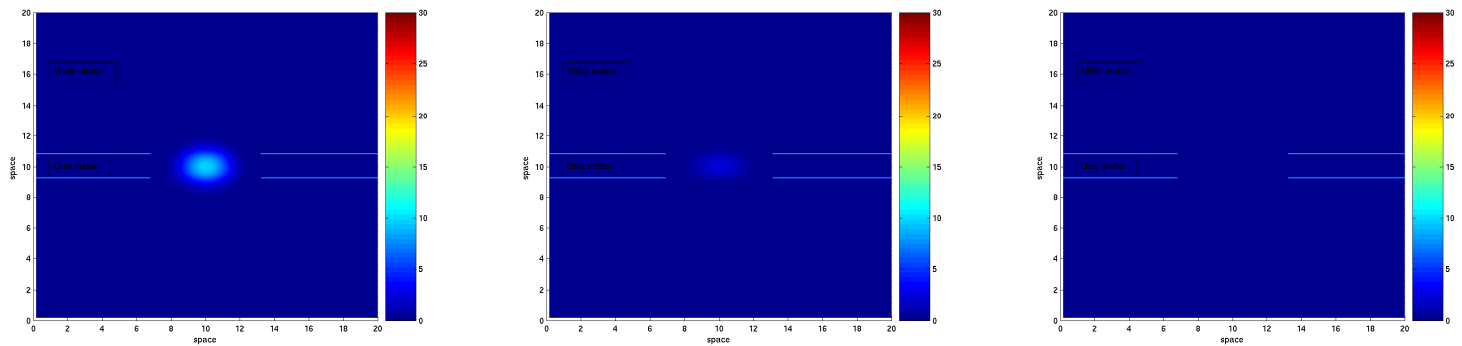

FiguRE 8. Extracellular potassium concentration after a $\mathrm{KCl}$ injection in the gray matter. Here the layer of gray matter is thin and the SD are not created.

\subsection{Influence of the geometry of the brain}

In this part, we will numerically study the effect of the geometry of the gray matter on the propagation of SD. Gridding a real map of a human brain is for the moment still difficult so here, the domain will be a cylinder which diameter is suddenly increased. It is impossible to use the fast Fourier transformation to solve the numerical system, so we used a finite elements method.

First, if the diameter of the cylinder is slightly increased, the SD can still spread through the entire brain (figure 9).

Now, if the diameter of the cylinder is largely increased, SD are stopped by this widening and can not spread through the entire brain (figure 10).

However, an important point is that the widening of the cylinder is very sudden. Hence, if it is just a little more progressive, the SD can pass through this widening (figure 11).

The stopping of SD by a sudden increase of the width of the gray matter may be an explanation for the non-observation of SD in the human brain. Indeed, even if the phenomena that create SD are the same as for 

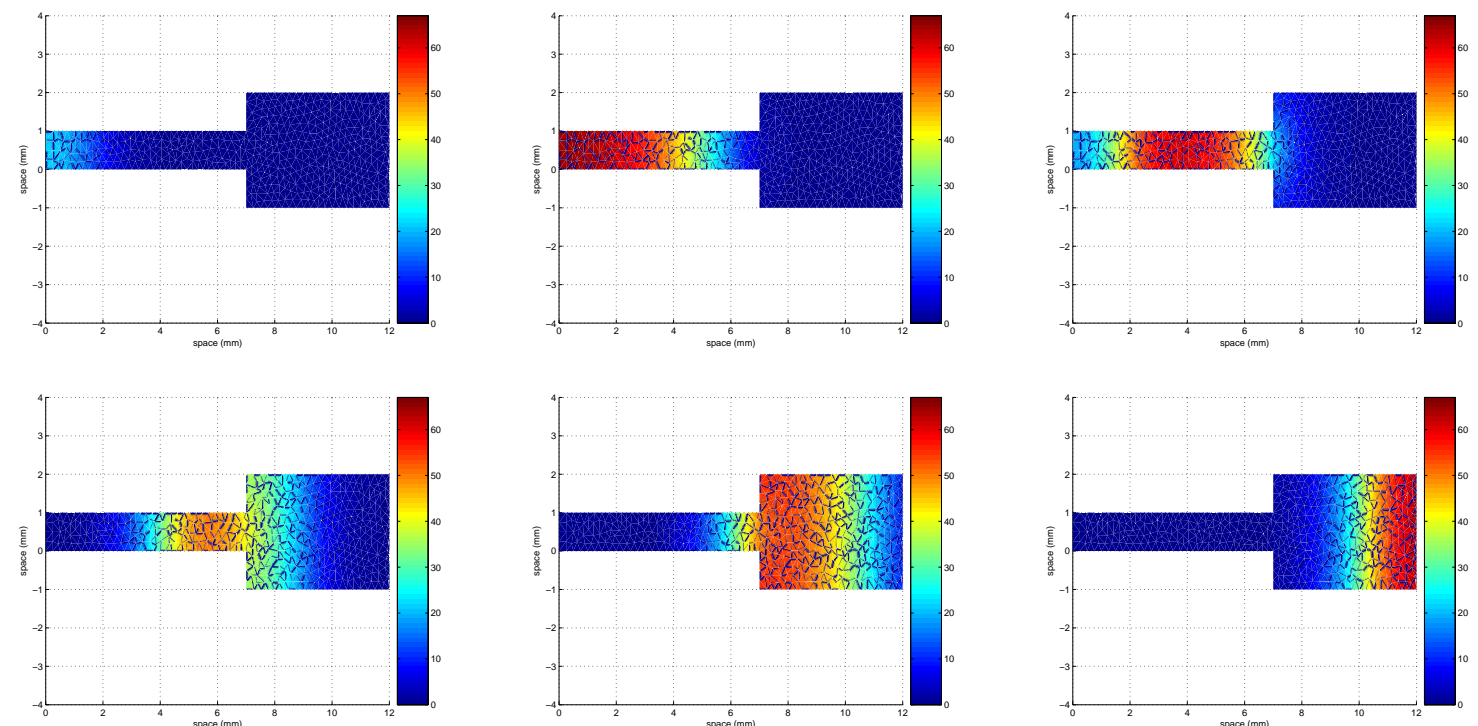

FiguRE 9. Extracellular potassium concentration after a $\mathrm{KCl}$ injection. The sudden widening of the cylinder does not stop the SD.
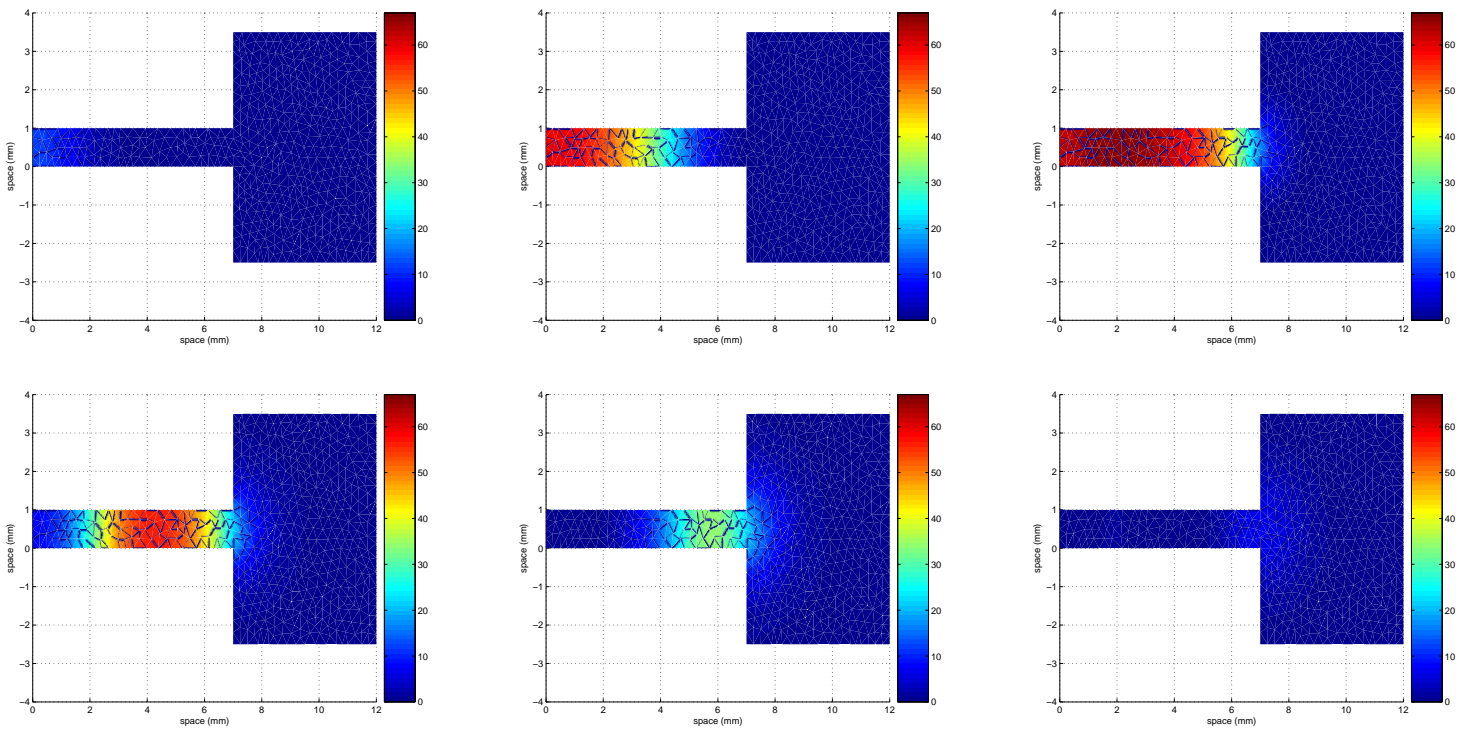

FiguRE 10. Extracellular potassium concentration after a $\mathrm{KCl}$ injection. The sudden widening of the cylinder stops the propagation of SD.

the rodent, the SD will never spread across a large area of the brain, they will always be stopped by the first important increase of the width of the gray matter (and these increases are very frequent in the human brain). This numerical study has been confirmed in [1]. 

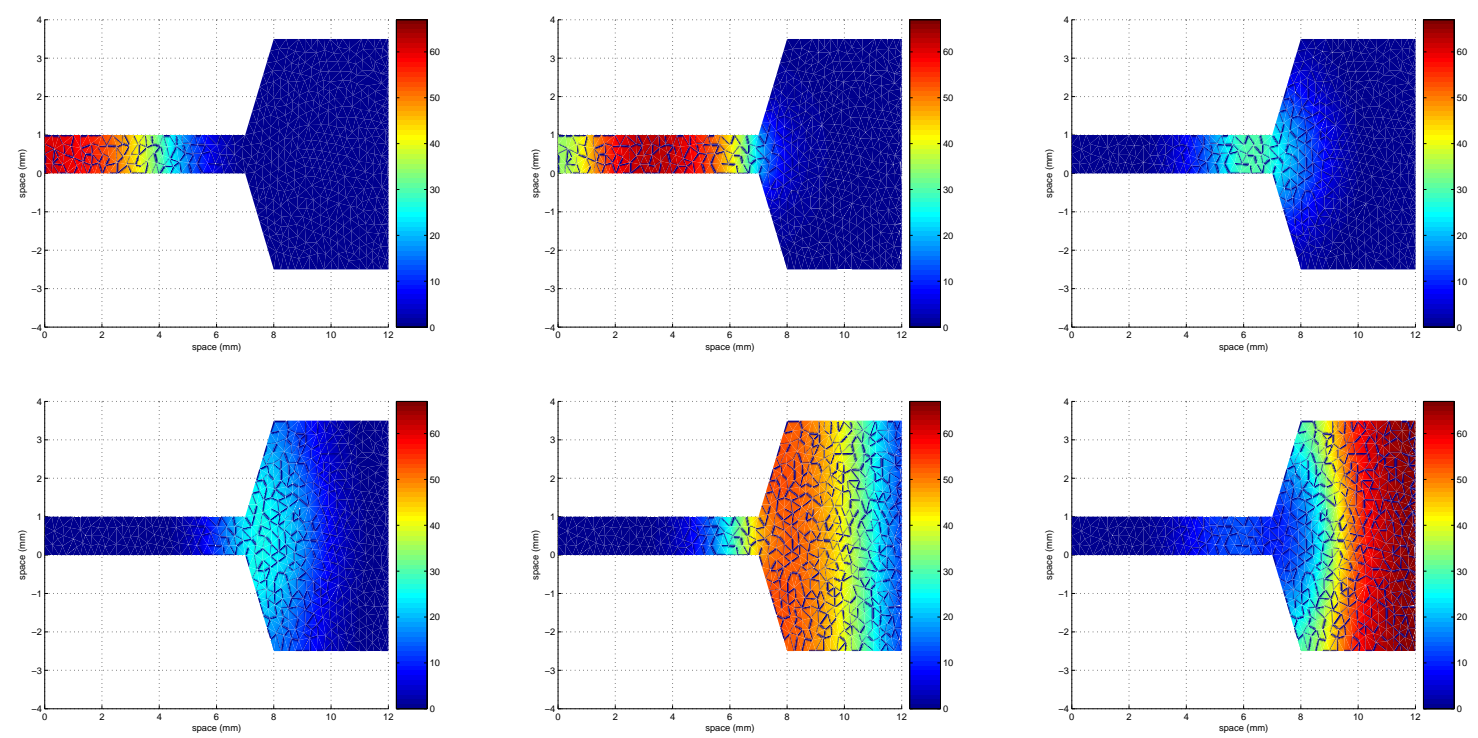

FiguRE 11. Extracellular potassium concentration after a $\mathrm{KCl}$ injection. The progressive widening of the cylinder does not stop the SD.

\section{Conclusion}

In this paper, we have detailed a model of spreading depressions. This model is centered around the ionic exchanges between neurons, astrocytes end extracellular space, particularly calcium and potassium. The model does not describe these phenomena down to the last detail, but in a phenomenological way.

A problem that we had to solve was to fix the values of all the parameters appearing in the mathematical system. The process that we have used takes a long time but gives results (It is almost impossible to find the parameters "by hand") and those results seem to be reliable.

With the parameters found with the random algorithm, this model of SD seems to globally fit the reality and we have used it to study the influence of the geometry of the brain and the influence of the white matter on the nonobservation of SD in human brain.

The next step will be to include this model in a global model of stroke. Thus we will be able to study numerically the influence of SD on the size of the infarct and even to numerically test the effects of some pharmacological agents.

Then we could also apply this model to a more realistic geometry of the brain, for the moment we are trying to get reliable images of the brain (with white and gray matters) and to grid them.

Finally this model could largely be improved by adding the effect of others ions and describing there movement in more detailed. In particular, we have planed to replace the simple ionic system by a model of ionic dynamic which is based on microscopic phenomena [7].

\section{REFERENCES}

[1] Chapuisat, G.; Grenier, E. Existence and nonexistence of traveling wave solutions for a bistable reaction-diffusion equation in an infinite cylinder whose diameter is suddenly increased. Commun. Partial Differ. Equations (2005) 30, No.10-12, 1805-1816

[2] Chapuisat, G. Existence and non-existence of curved front solution of a biological equation. Preprint.

[3] Chan, P.H. Cerebrovascular Disease 22nd Princeton Conference. Cambridge University Press, 2002.

[4] Duval, V.; Chabaud, S.; Girard, P.; Cucherat, M.; Hommel, M.; Boissel, J.P. Physiologically based model of acute ischemic stroke. Journal of Cerebral Blood Flow and Metabolism 2002, 22, 1010-1018. 
[5] Dronne, M.A.; Boissel, J.P.; Grenier, E.; Gilquin, H.; Cucherat, M.; Hommel, M.; Barbier, E.; Bricca, G. Mathematical modelling of an ischemic stroke: an integrative approach. Acta Biotheoritica 2004, 52, 255-272.

[6] Dronne, M.A.; Grenier, E.; Gilquin, H. Guidelines for inclusion of neuronal ionic exchange models in stroke models, preprint (2004).

[7] Dronne, M.A.; Boissel, J.P.; Grenier, E. A mathematical model of ion movements in grey matter during a stroke. J Theor Biol. 2005 Dec 17.

[8] Gorji, A.; Scheller, D.; Straub, H.; Tegtmeier, F.; Kohling, R.; Hohling, J.M.; Tuxhorn, I.; Ebner, A.; Wolf, P.; Werner Panneck, H.; Oppel, F.; Speckmann, E.J. Spreading depression in human neocortical slices. Brain Res 2001, 906 (1-2), 74-83.

[9] Leão, A.A.P. Spreading depression of activity in the cerebral cortex. J Neurophysiol, 1944, 10 (7), 359-390.

[10] Murray, J.D. Mathematical biology, Biomathematics; Springer-Verlag:Berlin, 1993.

[11] Shapiro, B. Osmotic forces and gap junctions in spreading depression: a computational model. J Comput Neurosci 2001, 10 (1), 99-120.

[12] Somjen, G. Ions in the Brain: Normal Function, Seizures, and Stroke. Oxford University Press: New York, 2004.

[13] Tuckwell, H.C.; Miura, R.M. A mathematical model for spreading cortical depression. Biophys J 1978, 23(2), $257-276$.

[14] Yi, C.S.; Fogelson, A.L.; Keener, J.P.; Peskin, C.S. A mathematical study of volume shifts and ionic concentration changes during ischemia and hypoxia. J Theor Biol 2003, 220 (1), 83-106. 